\title{
FONTES ALTERNATIVAS DE PÓLEN UTILIZADAS PELO BICUDO-DO- ALGODOEIRO EM DUAS REGIÕES PRODUTORAS DE ALGODÃO NA BAHIA ${ }^{1}$
}

\author{
JULIANA ALVES DE MACÊDO², MARIA APARECIDA CASTELLANI ${ }^{2 *}$, FRANCISCO DE ASSIS RIBEIRO DOS \\ SANTOS $^{3}$, PAULINO PEREIRA OLIVEIRA $^{3}$, RAQUEL PÉREZ MALUF ${ }^{2}$
}

\begin{abstract}
RESUMO - O bicudo-do-algodoeiro (Anthonomus grandis Boheman) (Coleoptera: Curculionidae) é um dos principais fatores limitantes para a expansão da cultura do algodão. Ele apresenta diferentes aspectos biológicos e comportamentais que garantem seu sucesso na exploração do agroecossistema algodoeiro. O objetivo deste trabalho foi identificar os recursos alimentares utilizados pelo bicudo na safra e entressafra de cultivos de algodão localizados nos municípios de Iuiu e Correntina (BA) e em ambientes de Caatinga e Cerrado, contíguos aos algodoais, respectivamente. As coletas dos adultos foram realizadas no período de fevereiro a setembro/2013 por meio de armadilhas com feromônio. As amostras foram processadas para identificação dos tipos polínicos encontrados no aparelho digestivo dos insetos. Os resultados indicaram que a praga explora 22 famílias botânicas como fonte de alimento, das quais 16 na Caatinga e 5 no Cerrado. Nos bicudos coletados em algodão foram encontrados 8 gêneros botânicos em Iuiu e 10 em Correntina, sendo apenas três coincidentes aos dois cultivos (Angelonia, Mimosa e Myrcia sp.1). Grãos de Pólen de 26 gêneros de plantas foram identificados para Caatinga e 6 para o Cerrado, ocorrendo três gêneros comuns às duas áreas (Myrcia sp.2, Eucalyptus e Mimosa). O bicudo explora fontes alternativas de alimento nas principais regiões produtoras de algodão da Bahia, com destaque para a Caatinga, que apresenta maior quantidade de famílias e gêneros botânicos utilizados pela praga.
\end{abstract}

Palavras chaves: Anthonomus grandis. Caatinga. Cerrado. Palinologia. Sobrevivência.

\section{ALTERNATIVE POLLEN SOURCES FOR BOLL WEEVIL IN TWO COTTON GROWING AREAS OF BAHIA STATE}

\begin{abstract}
Cotton boll weevil, Anthonomus grandis Boheman (Coleoptera: Curculionidae), is one of the most limiting factors for cotton crop expansion. It presents different biological and behavioral aspects that ensure its success on this agroecosystem. This research aimed to identify pollen sources used by this insect during harvest and intercrop periods of cotton cultivations in Iuiu and Correntina - BA, Brazil; moreover, evaluating Caatinga and Cerrado neighboring areas. Adult insect samplings were made from February to September of 2013, through pheromone traps. Samples were analyzed to identify the pollen types inside the digestive system of insects. The results indicated that the pest explores 22 botanical families as nourishment source, from which 16 in Caatinga and five in Cerrado. Regarding crop samples, eight genera were found in Iuiu and Correntina, being three found in both areas (Angelonia, Mimosa and Myrcia sp.1). Pollen from 26 genera were identified for Caatinga and six for Cerrado, with three genera in common for both (Myrcia sp.2, Eucalyptus, and Mimo$s a$ ). The boll weevil uses alternative sources of food within the main cotton growing regions in Bahia State, highlighting the Caatinga with most of the botanical families and genera explored by the pest.
\end{abstract}

Keywords: Anthonomus grandis. Caatinga. Cerrado. Palynology. Survival.

\footnotetext{
*Autor para correspondência

${ }^{1}$ Recebido para publicação em 28/04/2014; aceito em 06/05/2015.

Trabalho de Dissertação de Mestrado da primeira autora.

${ }^{2}$ Programa de Pós-Graduação em Agronomia (Fitotecnia), Universidade Estadual do Sudoeste da Bahia, C.P. 95, CEP: 45031-900, Vitória da Conquista (BA), Brasil; castellani@uesb.edu.br.

${ }^{3}$ Departamento de Ciências Biológicas, Universidade Estadual de Feira de Santana, CEP: 44036-900, Feira de Santana (BA), Brasil.
}

Revista Caatinga, Mossoró, v. 28, n. 3, p. 255 - 262, jul. - set., 2015 


\section{INTRODUÇ̃̃̃}

A cultura do algodão (Gossypium hirsutum L.) é uma das principais commodities agrícolas brasileiras e possui diversos produtos e subprodutos com demanda crescente no mundo, além de abrigar grande mão-de-obra em sua extensa cadeia produtiva. $\mathrm{O}$ Brasil se destaca no cenário mundial como o quinto maior produtor dessa cultura, o quarto lugar em exportação e o primeiro em produtividade em regime de sequeiro, sendo os estados do Mato Grosso e Bahia responsáveis por $83,2 \%$ da produção brasileira (REETZ et al., 2013).

A Bahia é o segundo maior produtor nacional de pluma e aparece no sétimo levantamento da safra 2013/2014 com 318,4 mil hectares plantados, um incremento $17,3 \%$ em relação ao ano agrícola anterior (CONAB, 2014), sendo a região Oeste do Estado responsável por cerca de $90 \%$ da produção baiana (ANUÁRIO ESTATÍSTICO DA BAHIA, 2014).

$\mathrm{O}$ bicudo Anthonomus grandis Boheman,1843 (Coleoptera: Curculionidae), é a principal praga das estruturas reprodutivas do algodão na América e se destaca pela ampla plasticidade fenotípica caracterizada pela alta variabilidade no tempo de desenvolvimento do ovo a adulto, ciclo de vida, multivoltinismo com gerações superpostas, capacidade de se alimentar de pólen de diversas famílias botânicas, capacidade de migração, dispersão (STADELER e BUTELER, 2007) e sobrevivência na entressafra. Após se alimentar e/ou ovipositar nos botões florais e também em maçãs quando em altas infestações o bicudo ocasiona a abscisão das estruturas reprodutivas. A queda dos botões florais configura-se nos maiores danos causados pelo bicudo e está relacionada, principalmente, com a presença de orifícios de oviposição (ALMEIDA; SILVA e RAMALHO, 2013). As infestações por esta praga independem do sistema de produção do algodão (TORRES et al., 2009). Em 2012 foram estimadas perdas médias causadas pelo bicudo da ordem de 70 a 130 US\$/ha (SORIA et al., 2013).

Identificar e compreender o comportamento e bioecologia desta praga é fundamental para a implementação de um programa de controle (GREENBERG et al., 2004). Sabe-se que durante a entressafra o inseto pode utilizar algumas estratégias de sobrevivência que possibilitam a manutenção de suas populações na safra seguinte, dentre elas a capacidade de se alimentar de pólen de muitas famílias de plantas, como relatado por diversos autores (CUADRADO e GARRALLA, 2000; CUADRADO, 2002; GABRIEL, 2002; GREENBERG et al., 2007; JONES, GREENBERG, EISCHEN, 2007; RIBEIRO et al., 2010).

Vários estudos têm sido desenvolvidos visando identificar hospedeiros de alimentação do bicudo. Na América do Sul destacam-se os trabalhos realizados na Argentina (CUADRADO; GARRALLA, 2000; CUADRADO, 2002) e, mais recentemente, no
Brasil (RIBEIRO et al., 2010), para as condições do Cerrado do Distrito Federal, demonstrando que o bicudo se utiliza de grande diversidade de pólen de plantas como alternativa ao pólen do algodão.

Para as condições do estado da Bahia há escassez de estudos ecológicos sobre o bicudo que possam subsidiar o desenvolvimento e/ou aperfeiçoamento das táticas de controle adequadas às diferentes regiões produtoras de algodão, localizadas em ambientes de Cerrado e de Caatinga.

O objetivo do presente trabalho foi identificar os recursos alimentares utilizados na safra e entressafra de algodão para as duas principais regiões produtoras da Bahia, instaladas em ambientes de Caatinga (Sudoeste) e Cerrado (Oeste).

\section{MATERIAL E MÉTODOS}

\section{Áreas de estudo}

O estudo foi conduzido nas regiões Sudoeste e Oeste da Bahia, abrangendo ambientes de Caatinga e Cerrado, respectivamente. Na região Sudoeste selecionou-se um cultivo de algodão de 16 ha (Latitude $14^{\circ} 16^{\prime} 710^{\prime \prime} \mathrm{Sul}$, Longitude $43^{\circ} 34^{\prime} 765^{\prime \prime}$ Oeste e Altitude: $841 \mathrm{~m}$ ), localizado no município de Iuiu, situado na Zona de depressão do Médio São Francisco, em ambiente de Caatinga, cuja fisionomia é denominada de Floresta Estacional Decidual, a qual ocorre em afloramento calcário, tanto neste ambiente quanto no Cerrado. O clima é semiárido, com precipitação anual variando de 500 a $800 \mathrm{~mm}$, e temperaturas médias anual de $26^{\circ} \mathrm{C}$ a $33^{\circ} \mathrm{C}$, de acordo com informações da Superintendência de Assuntos Econômicos e Sociais da Bahia (SEI, 1997). Foram utilizadas duas áreas experimentais, sendo área de algodão implantada em 08 de janeiro de 2013, na densidade de 12.820 plantas/ha, e outra de vegetação de Caatinga de aproximadamente 148 ha, distantes entre si 23 metros. $\mathrm{O}$ algodoal era circundado pela Rodovia BA160, que liga o município de Malhada a Bom Jesus da Lapa, pela Rodovia BA 030, que liga a Bahia ao estado de Minas Gerais, por outro cultivo de algodão e pela vegetação de Caatinga.

$\mathrm{Na}$ região Oeste selecionou-se um talhão de algodão comercial (Latitude 13 $45^{\prime} 705^{\prime \prime}$ Sul, Longitude $45^{\circ}$ 56' $163^{\prime}$ " Oeste e Altitude: 863), de 118 ha, implantado em 30 de dezembro de 2013 na Fazenda Tapera Grande, município de Correntina (BA), contígua a uma área de Cerrado de aproximadamente 2000 ha. A área cultivada estava separada da área de vegetação de Cerrado por uma estrada de $20 \mathrm{~m}$ de largura, apresentando no seu entorno apenas outros cultivos de algodão. A vegetação predominante da mata é caracterizada pela presença de árvores de porte baixo com troncos retorcidos com ramificações irregulares, arbustos e subarbustos que podem possuir xilopódios e órgãos subterrâneos os quais possibilitam a rebrota após a queima ou corte, sendo esta 
denominada de Cerrado sensu stricto, um dos vários componentes do mosaico das fitofisionomias do Cerrado (RIBEIRO e WALTER, 2008).

\section{Coleta dos Bicudos Adultos}

Nos cultivos de algodão, bem como nas vegetações de Caatinga e de Cerrado, foram delimitadas áreas experimentais de 6,72 ha para instalação das armadilhas e coleta dos bicudos adultos conforme metodologia estabelecida por Ribeiro et al. (2010). Em cada área foram demarcados quatro transectos de $280 \mathrm{~m}$ de comprimento, com $80 \mathrm{~m}$ de distância entre si. Em cada transecto foram instaladas cinco armadilhas equidistantes de $70 \mathrm{~m}$, denominadas linhas, constituindo ao todo cinco linhas por transecto, totalizando 20 armadilhas em cada área experimental.

As armadilhas utilizadas foram do tipo $\mathrm{Ac}$ countrap de cor verde fluorescente, usadas com Luretape $\mathrm{BW}-10$, que é um liberador impregnado com feromônio sintético do macho. A cor desta armadilha age sinergicamente com a isca de feromônio, aumentando a atratividade dos insetos, mesmo quando estes se encontram em número reduzido, sendo geralmente utilizadas para monitoramento da praga. Além do feromônio, as armadilhas receberam um fragmento de cerca de $3 \mathrm{~cm}$ de coleiras impregnadas com inseticida (Diazinon), com adição de novo fragmento a cada dois meses aproximadamente, visando ocasionar a morte dos insetos capturados e, consequentemente, interromper a digestão. Outrossim, as armadilhas foram instaladas sobre estacas de madeira ou de ferro a um metro da superfície do solo.

As armadilhas permaneceram no campo durante todo o período experimental com captura contínua dos bicudos e coleta dos mesmos a cada quinze dias. A substituição do feromônio foi realizada a cada 30 dias, segundo as recomendações do fabricante. Foram efetuadas 10 coletas nos meses de fevereiro, março, abril, maio e setembro, correspondendo a diferentes fases fenológicas da planta e da praga na cultura, a saber: a) antes do florescimento e colonização da cultura pelo bicudo: fevereiro em Iuiu e março em Correntina; b) durante o florescimento e estabelecimento da praga na cultura: abril para Iuiu e maio para Correntina; e c) após o florescimento (póscolheita) e fase de dispersão da praga para áreas de refúgio: setembro, apenas para as áreas de mata. As alterações nos meses de coleta entre as áreas experimentais ocorreram devido às diferenças nas épocas de plantio e no regime pluviométrico que interferiu no desenvolvimento das plantas, buscando-se trabalhar com o mesmo estádio fenológico do algodão.

Os insetos coletados foram acondicionados em sacos de papel, devidamente identificados quanto ao local e data e encaminhados ao Laboratório de Entomologia da Universidade Estadual do Sudoeste da Bahia para contagem, acondicionamento em frascos plásticos e armazenamento em freezer para pos- terior realização dos procedimentos de recuperação e identificação dos tipos polínicos.

\section{Identificação dos Recursos Alimentares}

Os bicudos coletados nas 20 armadilhas de cada área experimental e em cada época de coleta foram homogeneizados em uma única amostra para a retirada de subamostras de, no máximo, 25 bicudos, exceto para o mês de fevereiro na área de algodão próximo à Caatinga, pois a amostra única apresentava apenas cinco insetos e de março para as áreas de algodão e de Cerrado em Correntina, uma vez que a amostra única das duas últimas áreas possuía apenas quatro e dois bicudos, respectivamente. Assim, cento e oitenta e seis adultos foram preparados para as análises, procedendo-se de acordo com a metodologia descrita por Jones (2012). As etapas de maceração e acetólise (ERDTMAN, 1960) para as subamostras de fevereiro e março de todas as áreas experimentais foram realizadas com bicudos individualizados para posterior captura dos grãos de pólen e confecção das lâminas. Para as demais épocas, os bicudos de cada subamostra foram agrupados após dissecação, compondo uma única subamostra biológica para a confecção das lâminas.

O processamento palinológico do material foi realizado no Laboratório de Micromorfologia Vegetal (LAMIV) da Universidade Estadual de Feira de Santana (UEFS). Os tipos polínicos foram identificados em nível de família, gênero e espécie, quando possível utilizando-se, principalmente, as publicações de Salgado-Labouriau (1973), Barth (1989), Chavés et al. (1991), Roubik e Moreno (1991), Melhem et al. (2003), du Bocage et al. (2008) e Lima et al. (2008). Os respectivos nomes dos táxons seguiram a classificação mais recente do Angiosperm Phylogeny Group III (AGP III, 2009).

Os dados foram tabulados, considerando-se a presença e ausência dos tipos polínicos em função da procedência dos bicudos e da época das coletas. O grau de similaridade das áreas foi avaliado pelo índice de Jaccard, cuja fórmula é: $S J_{i j}=c /(a+b)-c$, em que: $a=$ número de famílias ocorrentes na área $1 ; b$ $=$ número de famílias ocorrentes na área 2 ; e $c=$ número de famílias comuns às duas áreas.

\section{RESULTADOS E DISCUSSÃO}

Considerando-se todas as áreas experimentais, 22 famílias botânicas foram encontradas a partir da recuperação dos grãos de pólen do trato digestivo dos bicudos, das quais 13 famílias em áreas de algodão e 17 nos ambientes de Caatinga e Cerrado, sendo 8 as famílias em comum para cultivos de algodão e os dois ambientes, quais sejam: Anacardiaceae; Fabaceae; Malvaceae; Melastomataceae; Mimosaceae; Myrtaceae; Plantaginaceae; Rubiaceae; e Solanaceae (Tabelas 1 e 2). 
Dos bicudos coletados nas áreas de algodão no presente estudo foram encontrados grãos de pólen de 13 famílias botânicas, sendo 8 em Iuiu e 10 em Correntina, sendo maior a diversidade na fase de colonização da cultura pelo bicudo, fevereiro e março para as duas áreas, respectivamente (Tabela 1). Isso era esperado, pois provavelmente grande parte dos bicudos coletados nas armadilhas era proveniente das vegetações próximas aos cultivos. Apenas quatro famílias foram comuns às duas áreas de algodão (Fabaceae, Myrtaceae, Plantaginaceae e Poaceae). Grãos de Pólen de malvácea foram encontrados apenas em bicudos da área de Correntina.

Em abril e maio, períodos correspondentes à estabilização da praga nos cultivos e auge do florescimento do algodoeiro, foram encontrados polén de apenas uma família para Iuiu (Fabaceae) e duas para Correntina (Fabaceae e Melastomataceae) (Tabela 1). O número reduzido de famílias foi esperado no algodão, mas esperava-se, também, que pólen de malvácea fosse encontrado nas duas áreas, o que não ocorreu. Uma das hipóteses para explicar a ausência de pólen de malvácea em bicudos coletados nas áreas de algodão está baseada na deterioração dos grãos de pólen do algodão nas amostras de bicudos estudadas, uma vez que o preparo de todas as amostras foi realizado em outubro de 2013. Grãos de pólen do algodoeiro são grandes, apresentando diâmetro variável de 70 a $120 \mu \mathrm{m}$ de diâmetro, com camadas externas (exina e intima) finas (SAAD, 1960), o que o torna frágil, podendo ser facilmente quebrado. Em alguns casos, o choque osmótico pode causar rompimento do grão de pólen. Por ser tão frágil, este efeito poderá causar a sua ruptura mais facilmente (JONES; GREENBERG, 2009). De acordo com Cate e Skinner (1978), os grãos de pólen permanecem no intestino dos bicudos por um período de 10 a 18 horas e fragmentos destes ficam retidos por até cinco dias após se alimentarem de botões florais do algodoeiro. Trabalhos realizados nos Estados Unidos, sob condições controladas em laboratório, mostraram que depois de decorridas 24 horas da ingestão do pólen de algodão por bicudos, os grãos de pólen recuperados, em sua maioria, encontravam-se muito fragmentados e a partir de 48 horas $100 \%$ dos grãos de pólen encontravam-se quebrados, sendo que às 120 horas apenas $1 \%$ dos bicudos tinha fragmento de pólen (JONES; GREENBERG, 2009).

Nas áreas de vegetação de Caatinga e de Cerrado a diversidade de famílias foi maior em relação aos algodoais, totalizando 17 nas duas áreas, das quais 16 ocorreram na Caatinga e cinco no Cerrado, sendo comuns às duas áreas as famílias Fabaceae e Myrtaceae (Tabela 2). Maior número de famílias foi obtido em fevereiro, para a Caatinga, e em maio, para o Cerrado (Tabela 2). Nas amostras do mês de fevereiro da área de Cerrado não foram encontrados grãos de pólen nas lâminas.

Foram identificados 29 gêneros e 11 espécies de plantas, confirmadas como hospedeiras de alimentação do bicudo, com possibilidades desse número ser maior para as duas categorias, pois ocorreram três tipos de Myrcia, dois de Psidium e quatro de Solanum (Tabelas 1 e 2). Dentre os hospedeiros identificados, observou-se a presença de várias espécies de plantas daninhas, plantas ornamentais e cultivadas nas duas regiões estudadas, além do algodão, plantas de importância agronômica e florestal como umbu, eucalipto e jacarandá, principalmente. Dentre as ornamentais, destacam-se o manacá ou quaresmeira, sabiá e sensitiva.

Para bicudos coletados nas áreas de algodão foram identificados 8 gêneros para Iuiu e 9 para Correntina, sendo apenas três coincidentes aos dois cultivos (Angelonia, Mimosa e Myrcia sp. 1) (Tabela 1). Para as áreas de vegetação não cultivada pólen de 26 gêneros foram identificados para Caatinga e 6 para o Cerrado, ocorrendo três gêneros comuns aos dois ambientes (Eucalyptus, Mimosa e Myrcia sp.2) (Tabela 2).

Há grande variação nos dados existentes na literatura sobre grãos de pólen tipos polínicos ingeridos por bicudos. Para as condições do sudeste do Texas e do nordeste do México foram identificados grãos de polén pertencentes a 15 famílias de plantas (BENEDICT et al.,1991), enquanto que no Mississipi (EUA) encontradas 82 famílias, 132 gêneros e 28 espécies, sendo as plantas da família Asteraceae (Compositae) os recursos mais explorados por este inseto (HARDEE; JONES e ADAMS, 1999). Na Argentina, Cuadrado e Garralla (2000) encontraram grãos de polén em bicudos procedentes de regiões produtoras de algodão associados às famílias Malvaceae, Compositae, Solanaceae, Euphorbiaceae, Amaranthaceae, Leguminosae (atualmente Fabaceae) e Polygonaceae. No Brasil, no estudo pioneiro de Ribeiro et al. (2010), foram encontrados pólen de 19 famílias em bicudos coletados no Distrito Federal, em áreas de algodão, Cerrado e Mata de Galeria, das quais nove (Poaceae, Asteraceae, Convolvulaceae, Fabaceae, Malvaceae, Malpighiaceae, Melastomataceae, Myrtaceae e Proteaceae) também ocorreram no presente trabalho e apenas cinco foram comuns às áreas de Cerrado dos dois trabalhos (Convouvulaceae, Fabaceae, Malvaceae, Myrtaceae e Poaceae). Esses dados indicam que mesmo dentro do Cerrado a qualidade dos hospedeiros de alimentação pode ser diferenciada. 
Tabela 1. Famílias, gêneros e espécies de plantas identificadas por meio de grãos de pólen recuperados do trato digestivo de Anthonomus grandis Boh. coletados em armadilhas de feromônio durante os meses de fevereiro, abril, março e maio em áreas de algodão. Iuiu e Correntina (BA), 2013.

\begin{tabular}{|c|c|c|c|c|c|}
\hline \multirow{3}{*}{ Família } & \multirow{3}{*}{ Tipos Polínicos } & \multicolumn{4}{|c|}{ Áreas de Algodão } \\
\hline & & \multicolumn{2}{|c|}{ Iuiu } & \multicolumn{2}{|c|}{ Correntina } \\
\hline & & Fev & Abr & Mar & Mai \\
\hline Anacardiaceae & Spondias tuberosa & $\mathrm{x}$ & & & \\
\hline Boraginaceae & Heliotropium & & & $\mathrm{x}$ & \\
\hline Convolvulaceae & & & & $\mathrm{x}$ & \\
\hline Fabaceae & Fabaceae tipo & & & & $\mathrm{x}$ \\
\hline Fabaceae & Calliandra & & & $\mathrm{x}$ & \\
\hline Fabaceae & & $\mathrm{x}$ & $\mathrm{x}$ & $\mathrm{x}$ & \\
\hline Fabaceae & Mimosa arenosa & & & $\mathrm{x}$ & \\
\hline Fabaceae & Mimosa tenuiflora & $\mathrm{x}$ & & & \\
\hline Malvaceae & Gossypium hirsutum & & & $\mathrm{x}$ & \\
\hline Melastomataceae & Tibouchina & & & $\mathrm{x}$ & $\mathrm{x}$ \\
\hline Myrtaceae & Eucalyptus & & & $\mathrm{x}$ & \\
\hline Myrtaceae & Myrcia sp.1 & $\mathrm{x}$ & & $\mathrm{x}$ & \\
\hline Myrtaceae & Myrcia sp.3 & $\mathrm{x}$ & & & \\
\hline Plantaginaceae & Angelonia & $\mathrm{x}$ & & $\mathrm{x}$ & \\
\hline Poaceae & & $\mathrm{x}$ & & $\mathrm{x}$ & \\
\hline Proteaceae & Roupala & $\mathrm{x}$ & & & \\
\hline Rubiaceae & Borreria & $\mathrm{x}$ & & & \\
\hline Rubiaceae & Richardia brasiliensis & $\mathrm{x}$ & & & \\
\hline Sapindaceae & & & & $\mathrm{x}$ & \\
\hline Solanaceae & Solanum sp.1 & $\mathrm{x}$ & & & \\
\hline Solanaceae & Solanum sp.3 & & & $\mathrm{x}$ & \\
\hline Solanaceae & Solanum sp.4 & & & $\mathrm{x}$ & \\
\hline $\mathrm{N}^{0}$ Total de Famílias: 13 & & & & & \\
\hline $\mathrm{N}^{0}$ de Famílias/Gêneros & & & & & \\
\hline
\end{tabular}

Tabela 2. Famílias, gêneros e espécies de plantas identificadas por meio de grãos de pólen recuperados do trato digestivo de Anthonomus grandis Boh. coletados em armadilhas de feromônio durante os meses de fevereiro, abril, maio e setembro em áreas de mata. Iuiu e Correntina (BA), 2013.

\begin{tabular}{|c|c|c|c|c|c|c|}
\hline \multirow{3}{*}{ Famílias } & \multirow{3}{*}{ Tipos de Polínicos } & \multicolumn{5}{|c|}{ Áreas de Mata } \\
\hline & & \multicolumn{3}{|c|}{ Caatinga } & \multicolumn{2}{|c|}{ Cerrado } \\
\hline & & Fev & Abr & Set & Mai & Set \\
\hline Amaranthaceae & Froelichia & & & & & $\mathrm{x}$ \\
\hline Anacardiaceae & Spondias tuberosa & & & $\mathrm{x}$ & & \\
\hline Anacardiaceae & Schinus terebinthifolius & & & $\mathrm{x}$ & & \\
\hline Apocynaceae & & & $\mathrm{x}$ & $\mathrm{x}$ & & \\
\hline Arecaceae & & & & & & $\mathrm{x}$ \\
\hline Arecaceae & Syagrus & $\mathrm{x}$ & & & & \\
\hline Asteraceae & Mikania & $\mathrm{x}$ & & & & \\
\hline Burseraceae & Protium & $\mathrm{x}$ & & & & \\
\hline Caesalpiniaceae & Chamaecrista & $\mathrm{x}$ & & & & \\
\hline Fabaceae & Fabaceae type & $\mathrm{x}$ & & & $\mathrm{x}$ & \\
\hline Fabaceae & Machaerium & $\mathrm{x}$ & & & & \\
\hline Fabaceae & & $\mathrm{x}$ & & & & \\
\hline Fabaceae & Mimosa arenosa & $\mathrm{x}$ & & $\mathrm{x}$ & & \\
\hline Fabaceae & Mimosa caesalpinifolia & $\mathrm{x}$ & $\mathrm{x}$ & & & \\
\hline Fabaceae & Mimosa sensitive & & & & $\mathrm{x}$ & \\
\hline Fabaceae & Mimosa tenuiflora & & & $\mathrm{x}$ & $\mathrm{x}$ & $\mathrm{x}$ \\
\hline
\end{tabular}

Revista Caatinga, Mossoró, v. 28, n. 3, p. 255 - 262, jul. - set., 2015 
Tabela 2. Continuação.

\begin{tabular}{|c|c|c|c|c|c|c|}
\hline \multirow{3}{*}{ Famílias } & \multirow{3}{*}{ Tipos de Polínicos } & \multicolumn{5}{|c|}{ Áreas de Mata } \\
\hline & & \multicolumn{3}{|c|}{ Caatinga } & \multicolumn{2}{|c|}{ Cerrado } \\
\hline & & Fev & Abr & Set & Mai & Set \\
\hline Fabaceae & Piptadenia stipulacea & $\mathrm{x}$ & & & & \\
\hline Fabaceae & Plathymenia & $\mathrm{x}$ & & & & \\
\hline Fabaceae & Plathymenia reticulata & $\mathrm{x}$ & & & & \\
\hline Lamiaceae & Hyptis & $\mathrm{x}$ & & & & \\
\hline Malphigiaceae & Byrsonima & $\mathrm{x}$ & & & & \\
\hline Malpighiaceae & Malpighiacae Tipo & $\mathrm{x}$ & & & & \\
\hline Malvaceae & Gossypium hirsutum & & & $\mathrm{x}$ & & \\
\hline Melastomataceae & Miconia & $\mathrm{x}$ & & & & \\
\hline Melastomataceae & Schwackea & & & & $\mathrm{x}$ & \\
\hline Melastomataceae & Tibouchina & $\mathrm{x}$ & & & & \\
\hline Myrtaceae & Eucalyptus & $\mathrm{x}$ & & & & $\mathrm{x}$ \\
\hline Myrtaceae & Myrcia sp.1 & $\mathrm{x}$ & & & & \\
\hline Myrtaceae & Myrcia sp.2 & $\mathrm{x}$ & & & & $\mathrm{x}$ \\
\hline Myrtaceae & Psidium sp.1 & $\mathrm{x}$ & & & & \\
\hline Myrtaceae & Psidium sp.2 & & & & $\mathrm{x}$ & \\
\hline Plantaginaceae & Angelonia & $\mathrm{x}$ & & & & \\
\hline Rubiaceae & Borreria & $\mathrm{x}$ & & & & \\
\hline Rubiaceae & Richardia scabra & $\mathrm{x}$ & & & & \\
\hline Rubiaceae & Mitracarpus tipo & $\mathrm{x}$ & & & & \\
\hline Rutaceae & Citrus & $\mathrm{x}$ & & & & \\
\hline Solanaceae & Solanum sp.1 & $\mathrm{x}$ & & & & \\
\hline Solanaceae & Solanum $\mathrm{n}^{\circ} 2$ & $\mathrm{x}$ & & & & \\
\hline \multicolumn{2}{|c|}{$\mathrm{N}^{0}$ de famílias Total $=17$} & \multicolumn{3}{|c|}{-} & \multicolumn{2}{|c|}{-} \\
\hline \multicolumn{2}{|c|}{ Número de Famílias/Gêneros } & \multicolumn{3}{|c|}{$16 / 26$} & \multicolumn{2}{|c|}{$5 / 6$} \\
\hline
\end{tabular}

Os índices de similaridade referentes aos recursos polínicos explorados pelo bicudo nas áreas confrontadas variaram de 0,00 a 0,31 (Tabela 3), sendo considerados nulos ou baixos, pois de acordo com Kent e Coker (1992) apenas valores maiores ou iguais a 0,5 indicam alta similaridade. De modo geral, esses resultados eram esperados devido aos diferentes ambientes que predominam nas duas áreas de estudo, Caatinga em Iuiu e Cerrado em Correntina, com composição florística distinta.

Tabela 3. Índice de Similaridade de Jaccard para as áreas de coleta de bicudos para análise dos grãos de pólen, em Iuiu e Correntina (BA), 2013.

\begin{tabular}{lcc}
\hline \multicolumn{1}{c}{ Áreas Confrontadas } & Época & J \\
\hline Algodão Iuiu x Algodão Correntina & Fevereiro x Março & 0,28 \\
Caatinga x Cerrado & Setembro x Setembro x Setembro & 0,14 \\
Caatinga x Cerrado & Abril x Maio & 0,00 \\
Algodão Iuiu x Caatinga & Fevereiro x Fevereiro & 0,31 \\
\hline
\end{tabular}

O ambiente de Caatinga está predominante no Nordeste brasileiro, caracterizado pela grande extensão, diferentes tipos de clima e de solo e multiplicidade nas formas de relevo do semiárido, resultando em diferentes paisagens que derivam em alto grau de variabilidade da flora (SANTANA; SOUTO, 2006). Alguns gêneros e espécies de plantas identificados no presente trabalho são típicos de Caatinga e já foram registrados em estudos realizados em Jacobina e Senhor do Bonfim (BA), a exemplo da aroeira, umbuzeiro, licuri, jurema preta, jurema branca (RAMALHO et al., 2009).

Os dados do presente trabalho evidenciam a importância e, ao mesmo tempo, as diferenças entre os dois ambientes estudados na ecologia do bicudo- do-algodoeiro, sendo fundamentais para a sobrevivência da praga, praticamente o ano todo. Considera -se a necessidade de ampliar os estudos sobre os hábitos alimentares do bicudo, especialmente na Caatinga, que englobem aspectos qualitativos e quantitativos dos tipos polínicos que possam subsidiar o aperfeiçoamento de estratégias de controle cultural da praga.

\section{CONCLUSÕES}

O bicudo-do-algodoeiro explora fontes alternativas de alimento nas principais regiões produto- 
ras de algodão da Bahia, com destaque para a Caatinga, que apresenta maior quantidade de famílias e gêneros botânicos utilizados pela praga.

$\mathrm{O}$ uso de fontes alternativas de alimento constitui em estratégia importante de sobrevivência da praga na entressafra da cultura do algodão nas regiões produtoras da Bahia.

\section{REFERÊNCIAS}

APG III. An update of the Angiosperm Phylogeny Group classification for the orders and families of flowering plants: APG III. Botanical Journal of the Linnean Society, London, v. 161, n.2, p. 105-121, 2009.

ALMEIDA, R. P. de; SILVA, C. A.; RAMALHO, F. de S. Manejo Integrado de Pragas do Algodoeiro no Brasil. 59 p., 2013. Disponível em: http:// www.cnpa.embrapa.br/aunidade/

MIP_algodoeiro_2013.pdf. Acesso em 12 mar. 2015.

ANUÁRIO ESTATÍSTICO DA BAHIA. Salvador, v. $27,2014.696$ p.

BARTH, O. M. O pólen no mel brasileiro. Rio de Janeiro: Gráfica Luxor, 1989. 151p.

BENEDICT, J.H. et al. Pollens ingested by boll weevils (Coleoptera: Curculionidae) in southern Texas and northeastern Mexico. Journal of Economic Entomology, Annapolis, v. 84, n.1, p. 126-131, 1991.

CATE, J.R.; SKINNER, J.L. Fate and identification of pollen in the alimentary canal of the boll weevil. Southwestern Entomologist, Washington, v. 3, n.4, p. 263-265. 1978.

CHÁVEZ, R. P.; WIECHERS, B. L.; VILLANUEVA, G. R. Flora palinologica de la reserva de la biosfera de SianKa'An Quintana Rôo, México. Chetumal: Centro de Investigaciones Quintana Roo, 1991. 321p.

CONAB (Companhia Nacional de Abastecimento). Acompanhamento de safra brasileira: grãos, safra 2013-2014, Sétimo levantamento. Brasília, DF: Companhia Nacional de Abastecimento, 2014. 41p.

CUADRADO, G. A. Anthonomus grandis Boheman (Coleoptera: Curculionidae) en la Zona Central y Sur Oeste de Misiones, Argentina: polen como fuente alimenticia y su relacioncom o estado fsiológico en insectos adultos. Neotropical Entomology, Londrina, v. 31, n.1, p. 121-132, 2002.

CUADRADO, G. A.; GARRALLA, S. S. Plantas alimentícias alternativas del picudo de algodonero
(Anthonoumus grandis Boh.) (Coleoptera: Curculionidae) en la Provincia de Formosa, Argentina. Análispalinológicodeltracto digestivo. Anais da Sociedade Entomológica do Brasil, Londrina, v. 29 , n.2, p. $245-255,2000$.

DU BOCAGE, A.L.; SOUZA, M.A., T.S.; GONÇALVES-ESTEVES, V. Palinotaxonomia de espécies de Acacia (Leguminosae - Mimosoideae) no Semi-Árido brasileiro. Rodriguésia, Rio de Janeiro, v. 59, n. 3, p. 587-596, 2008.

ERDTMAN, G. The acetolysis method. A revised description. Svensk Botanisk Tidskrift, Stockholm, v. 39, p. 561-564, 1960.

GABRIEL, D. Longevidade do bicudo do algodoeiro Anthonomus grandis Boh., criado em hospedeiras alternativas no laboratório. Arquivos do Instituto Biológico, São Paulo, v. 69, n.3, p. 123-126, 2002.

GREENBERG, S. M. et al. Influence of different cotton fruit sizes on bol weevil (Coleoptera: Curculionidae) oviposition and survival to adulthood. Environmental Entomology, Annapolis, v. 33, n.2, p. 443-449, 2004

GREENBERG, S.M. et al. Survival of boll weevil (Coleoptera: Curculionidae) adults after feeding on pollens from various sources. Insect Science, Beijing, v. 14, n.6, p. 503-510, 2007.

HARDEE, D. D.; JONES, G. D.; ADAMS, L. C. Emergence, movement, and host plants of boll weevils (Coleoptera: Curculionidae) in the Delta of the Mississippi. Journal of Economic Entomology, Annapolis, v. 92, n.1, p. 130-139, 1999.

JONES, G. D. Pollen Extraction from Insects. Palynology, London, v. 36, n.1, p. 86-109, 2012.

JONES, G. D.; GREENBERG, S.M. Cotton pollen retention in the boll weevils: a laboratory experiment. Palynology, London, v. 33, n.1, p. 157-165, 2009.

JONES, G. D.; GREENBERG, S. M.; EISCHEN, F. A. Almond, melon and pigweed pollen retention in the boll weevil (Coleoptera; Curculionidae). Palynology, London, v. 31, n.1, p.81-93, 2007.

KENT, M.; COKER, P. Vegetation description analyses. Behaven Press: London. 1992, 363p.

LIMA, L. C. L.; SILVA, F. H. M.; SANTOS, F. A. R. Palinologia de espécies de Mimosa L. (Leguminosae - Mimosoideae) do Semi-Árido brasileiro. Acta Botanica Brasilica, São Paulo, v. 22, n. 3, p. 794-805, 2008. 
MELHEM, T. S. et al. Variabilidade polínica em plantas de Campos do Jordão (São Paulo, Brasil). Boletim do Instituto de Botânica, São Paulo, n.16, p. 1-104, 2003.

RAMALHO, C. I. et al. Flora Arbóreo-Arbustiva em Áreas de Caatinga no Semiárido Baiano, Brasil. Caatinga, Mossoró, v. 22, n.3, p. 182-190, 2009.

REETZ, E. R. et al. Anuário Brasileiro de Algodão. Santa Cruz do Sul, RS: Gazeta, 2013. 144 p.

RIBEIRO, J. F.; WALTER, B. M. T. As principais fitofisionomias do Cerrado. In: SANO, S. M.; ALMEIDA. S. P.; RIBEIRO, J. F. (Ed.). Cerrado: ecologia e flora. Planaltina: Embrapa Cerrados, 2008. p.153-212.

RIBEIRO, P. A. et al. Alternative food sources and overwintering feeding behavior of the boll weevil, Anthonomus grandis Boheman, (Coleoptera: Curculionidae) under the tropical conditions of Central Brasil. Neotropical Entomology, Londrina, v. 39, n.1, p. 28-34, 2010.

ROUBIK, D. W.; MORENO, P. J. E. Pollen and spores of Barro Colorado Island. St. Louis: Missouri Botanical Garden, 1991. 268p. (Monographs in Systematic Botany).

SAAD, S. I. The sporoderm stratification in the Malvaceae. Pollen et Spores, Paris, v. 1, n.2, p. 1341, 1960 .

SALGADO-LABOURIAU, M. L. Contribuição à palinologia dos cerrados. Rio de Janeiro, RJ: Academia Brasileira de Ciências, 1973. 273 p.

SANTANA, J. A. da S.; SOUTO, J. S. Diversidade e Estrutura Fitossociológica da Caatinga na Estação Ecológica do Seridó-RN. Revista de Biologia e Ciências da Terra, Campina Grande, v. 6, n.2, p. 232242, 2006.

SORIA, M. F. et al. Alerta para o bicudo-doalgodoeiro - breve panorama pré-safra 2012/2013 e ações para o combate da praga. Circular Técnica, Instituto Matogrossense do Algodão, n. 3, 2013. 4 p.

STADLER, T.; BUTELER, M. Migration and dispersal of Anthonomus grandis (Coleoptera: Curculionidae) in South America. Sociedad Entomológica Argentina, Buenos Aires, v. 66, n.3-4, p. 205-217, 2007.

SUPERINTENDÊNCIA DE ESTUDOS ECONÔMICOS E SOCIAIS DA BAHIA (SEI, BA). Anuário de Estatística do Estado da Bahia. Salvador, BA, $1997.687 \mathrm{p}$.
TORRES, J. B.; RUBERSON, J. R.; WHITEHOUSE, M. Transgenic cotton for sustainable pest management. In E. Lichtfouse (ed.), Sustainable Agriculture Reviews: organic farming, pest control and remediation of soil pollutants. New York: Springer, 2009. 418p. 